BULLETIN OF THE

AMERICAN MATHEMATICAL SOCIETY

Volume 81, Number 1, January 1975

\title{
ALGEBRAS OF ANALYTIC FUNCTIONS ON DEGENERATING RIEMANN SURFACES
}

\author{
BY RICHARD ROCHBERG ${ }^{1}$
}

Communicated by Richard Goldberg, September 30, 1974

I. Introduction. By a Riemann surface we mean a finite bordered Riemann surface. For a Riemann surface $S$ denoted by $A(S)$ the supremum normed Banach algebra of functions continuous on $S$ and analytic on the interior of $S$. For any two Banach spaces $A$ and $B$ define $d(A, B)=$ $\log \inf \left\{\|T\|\left\|T^{-1}\right\| ; T\right.$ a continuous invertible linear map of $A$ onto $\left.B\right\}$. For $S_{1}$ and $S_{2}$ homeomorphic Riemann surfaces define $d\left(S_{1}, S_{2}\right)=$ $d\left(A\left(S_{1}\right), A\left(S_{2}\right)\right)$. It is known [7] that $d$ defines a metric on $R\left(S_{1}\right)$, the Riemann space of $S_{1}$, the space of conformal equivalence classes of Riemann surfaces homeomorphic to $S_{1}$, and that the topology induced by this metric is the same as that induced by the Teichmüler metric. The metric space $\left(R\left(S_{1}\right), d\right)$ is not complete. In this note we present properties of the ideal elements that are introduced in forming the completion of the metric space $\left(R\left(S_{1}\right), d\right)$. Proofs of these and related results will appear in a later publication.

The main result is that the new elements are connected degenerate Riemann surfaces. In fact, the results presented strongly suggest (but do not prove) that the completion of $\left(R\left(S_{1}\right), d\right)$ is formed by adjoining to $R\left(S_{1}\right)$ exactly those elements obtained by "pinching to a point" of closed noncontractible curves on surfaces in $R\left(S_{1}\right)$.

On an informal geometric level these results are related to results on degeneration of compact surfaces [4] and results on boundary points of Teichmüller space $[1],[2],[5]$.

II. An example. The following example illustrates many of the phenomena described in Theorem 2. For $0<r<1$ let $S_{r}=\{r \leqslant|z| \leqslant 1\}$. Let $A_{r}=A\left(S_{r}\right)$. Let $S_{0}$ be two closed disks with their centers identified, and let $A_{0}$ be the algebra of continuous functions on $S_{0}$ which are "analytic" on the interior.

AMS (MOS) subject classifications (1970). Primary 30A98, 46J15, $32 \mathrm{G} 15$.

${ }^{1}$ This research supported in part by NSF grant GP 34628.

Copyright $\odot$ 1975, American Mathematical Society 
THEOREM 1. The set $\left\{A_{r}\right\}_{0<r<1}$ is a complete metric space with respect to the metric $d$. This space is homeomorphic (in the natural way) to $[0,1)$.

This example contains a great deal of negative information about the metric $d$.

COROllary. The metric space $\left(R\left(S_{1 / 2}\right), d\right)$ is not complete. Hence the metric $d$ on $R\left(S_{1 / 2}\right)$ is not equivalent to the Teichmüller metric.

Corollary. Given $\epsilon$ positive one can find Banach algebras $A$ and $B$ with maximal ideal spaces $M(A)$ and $M(B)$ such that $d(A, B)<\epsilon$, but the homology groups of $M(A)$ and $M(B)$ are not isomorphic.

This example is best understood by thinking of the surfaces $S_{r}$ for small $r$ as being obtained by letting the length of the generator of the homology group tend to zero. To make this precise we need a notion of the length of a homology class.

III. Lengths of cycles. The appropriate notion of length in this context is that of "harmonic length" introduced by Landau and Osserman [3].

Let $S$ be a Riemann surface and $\gamma$ a smooth closed curve on $S$. The harmonic length of $\gamma, l(\gamma)$ is defined by $l(\gamma)=\sup \left\{\int_{\gamma} * d u ; u\right.$ a real harmonic function on $S$, $\left.\sup _{S}|u| \leqslant 1\right\}$. (*du is the harmonic conjugate of the differential du.) $l(\gamma)$ is seen to depend only on the homology class of $\gamma$. One easily checks that if $\gamma_{r}$ is a generator of the integer homology group of the surfaces $S_{r}$ in the previous example then $l\left(\gamma_{r}\right) \rightarrow 0$ as $r \rightarrow 0$.

Let $S_{1}, S_{2}, \cdots$ be a sequence of homeomorphic Riemann surfaces. Let

$$
h\left(\left\{S_{n}\right\}\right)=\lim _{\epsilon \rightarrow 0} \varlimsup_{n \rightarrow \infty} h_{\epsilon}\left(S_{n}\right)
$$

where $h_{\epsilon}\left(S_{n}\right)$ is the dimension of the span in $H_{1}\left(S_{n}, R\right)$ of the set of $\gamma$ in $H_{1}\left(S_{n}, Z\right)$ with $l(\gamma)<\epsilon$. Thus $h\left(\left\{S_{n}\right\}\right)$ measures the number of linearly independent homology classes which are being pinched as $n$ becomes large.

IV. The main result. By a degenerate Riemann surface we mean the object obtained from a possibly disconnected Riemann surface by making finitely many identifications of finite point sets. Let $S_{1}$ be a Riemann surface of genus $g$ which has $k$ boundary contours.

THEOREM 2. Let $S_{1}, S_{2}, \cdots$ be a sequence of Riemann surfaces homeomorphic to $S_{1}$ such that $\left\{S_{n}\right\}$ is a Cauchy sequence in $\left(R\left(S_{1}\right), d\right)$. 
There is a Banach space $A_{\infty}$ such that $d\left(A_{\infty}, A\left(S_{n}\right)\right) \rightarrow 0$ as $n \rightarrow \infty . A_{\infty}$ has the following additional properties. $A_{\infty}$ is a point separating algebra of continuous boundary value analytic functions on the connected degenerate Riemann surface $S_{\infty}$ (= maximal ideal space of $\left.A_{\infty}\right)$. $\partial S_{\infty}$, the boundary of $S_{\infty}$, consists of $k$ circles. The closure of the space of real parts of functions in $A_{\infty}$ in the space of real valued continuous functions on $\partial S_{\infty}$ has codimension $2 g+k-1$. The dimension of the homology group $H_{1}\left(S_{\infty}, \mathbf{R}\right)$ is $2 g+k-1-h\left(\left\{S_{n}\right\}\right)$.

The proof uses results on almost isometries of function algebras [6] to construct the algebra $A_{\infty}$ and then uses techniques from the theory of function algebras to describe the maximal ideal space of $A_{\infty}$.

One consequence of this result is that if no cycles are pinched to zero then the surfaces are not degenerating.

COROLlaRY. If $h\left(\left\{S_{n}\right\}\right)=0$ then $S_{\infty}$ is a Riemann surface homeomorphic to $S_{1}$.

V. Another example. Let $S$ be a compact Riemann surface of genus 1 . Let $p$ be a point of $S$. One can choose a local uniformizer $w$ at $p$ so that the surfaces $S_{n}=\{q \in S \mid w(q) \geqslant 1 / n\}, n=1,2, \cdots$, satisfy the hypotheses of Theorem 2. In this case the algebra $A_{\infty}$ will be the algebra of all functions $f$ which are continuous on $\{|z| \leqslant 1\}$, analytic in $\{|z|<1\}$, and satisfy $f^{\prime}(0)=0$.

\section{REFERENCES}

1. L. Bers, Spaces of degenerating Riemann surfaces, discontinuous groups and Riemann surfaces, Princeton Univ. Press, Princeton, N. J., 1974.

2. - On the boundaries of Teichmüller spaces and on kleinian groups. I, Ann. of Math. (2) 91 (1970), 570-600. MR 45 \#7044.

3. H. J. Landau and R. Osserman, On analytic mappings of Riemann surfaces, J. Analyse Math. 7 (1959/60), 249-279. MR 23 \#A311.

4. A. Lebowitz, On the degeneration of Riemann surfaces, Advances in the Theory of Riemann Surfaces (Proc. Conf., Stony Brook, N. Y., 1969), Ann. of Math. Studies, no. 66, Princeton Univ. Press, Princeton, N. J., 1971, pp. 265-286. MR 43 \#7622.

5. B. Maskit, On the boundaries of Teichmüller spaces and on klienian groups. II, Ann. of Math. (2) 91 (1970), 607-639. MR 45 \#7045.

6. R. Rochberg, Almost isometries of Banach spaces and moduli of planar domains, Pacific J. Math. 49 (1973), 445-466.

7. Almost isometries of Banach spaces and moduli of Riemann surfaces. II, Duke Math. J. (to appear)

DEPARTMENT OF MATHEMATICS, WASHINGTON UNIVERSITY, ST. LOUIS, MISSOURI 63130 